\title{
Stochastic fluctuations of persistent currents in mesoscopic rings
}

\author{
Ulrich Eckern ${ }^{1}$ and Albert Schmid ${ }^{2}$ \\ 'Kernforschungszentrum Karlsruhe, Institut für Nukleare Festkörperphysik, Postfach 3640, \\ W-7500 Karlsruhe 1, Germany \\ ${ }^{2}$ Institut für Theorie der Kondensierten Materie der Universität Karlsruhe, Postfach 6980, \\ W-7500 Karlsruhe 1, Germany
}

\begin{abstract}
We investigate systematically the effect of the electronic Coulomb interaction on the stochastic fluctuations (from sample to sample) in the persistent currents $I(\phi)$ of mesoscopic rings threaded by a magnetic flux $\phi$. In contrast to our recent publication [Europhys. Lett. 18 (1992) 457], we avoid separation of impurity and interaction scattering events and we present here a comprehensive view. By this alternative procedure, we are able to confirm our earlier result, namely that $\left(I^{2}\right\rangle^{1 / 2} \sim$ $e v_{F} / L$, which is a much larger quantity than the average current $\left\langle n-e v_{F} l / L^{2}\right.$.
\end{abstract}

Keywords: Weak localization; Fermion systems; Metals.

\section{General theory}

Following the seminal paper of Büttiker, Imry and Landauer [1], the phenomenon of persistent currents in mesoscopic samples has been discussed theoretically in various papers [2-11]. Accordingly, a normal-metal ring threaded by a magnetic flux $\phi$ should carry an equilibrium current; in other words, a magnetic moment should appear as a consequence of the applied flux. Most important in the development of this field have been the experimental investigations of the two groups at Bell Labs [12] and at IBM [13], where flux dependent persistent currents have been shown to exist in mesoscopic metal rings.

For sake of simplicity, we consider in the following an idealized situation where the width of the metal ring is so small (compared to the circumference $L$ ) that magnetic field penetration into the metal can be neglected. In the experiments [12,13], the situation is not so ideal; therefore, we will discuss the necessary corrections to the idealized theory at the end.

Without magnetic field penetration, the energy and the thermodynamic potential $K(\phi)$ of a given sample depend only on the magnetic flux. As the persistent current $I(\phi)$ is an equilibrium phenomenon, it can be calculated [6] by taking the derivative according to

$$
I(\phi)=-c \frac{\partial K(\phi)}{\partial \phi}
$$


Depending on the variables to be kept constant in the experiment

$$
K(\phi) \rightarrow \begin{cases}F(N, \phi) & \mathrm{CE} \\ \Omega(\mu, \phi) & \mathrm{GCE}\end{cases}
$$

for the canonical and the grand canonical ensemble, respectively.

In the case of time reversal symmetry, $K(\phi)$ is an even function of $\phi$; and in the idealized situation, it is also periodic in $\phi$ with primitive period

$$
\phi_{0}=2 \pi \hbar c / e
$$

Then, it follows that

$$
\begin{aligned}
& I(-\phi)=-I(\phi) \\
& I\left(\phi+\phi_{0}\right)=I(\phi)
\end{aligned}
$$

for any realization of the impurity positions, and that the following Fourier decomposition is valid: ${ }^{1}$

$$
\begin{aligned}
& I(\phi)=\sum_{m=1}^{\infty} I_{m} \sin 2 \pi m \phi / \phi_{0} \\
& K(\phi)=\sum_{m=1}^{\infty} K_{m} \cos 2 \pi m \phi / \phi_{0} .
\end{aligned}
$$

In the following, we put $\hbar=c=k_{B}=1$. Then $\phi_{0}=2 \pi / e$, and the following relation holds:

$$
I_{m}=e m K_{m}
$$

For sake of completeness, we remark that the persistent current generates a magnetic moment

$$
M(\phi)=\pi(L / 2 \pi)^{2} I(\phi)
$$

with $L$ being the circumference of the ring.

\section{Correlation functions and experimental results}

It is known that the properties ${ }^{2}$ of mesoscopic samples depend sensitively on the actual impurity configuration. Therefore, it is appropriate to introduce an ensemble of

1 See also Ref. [10].

2 A theoretical and an experimental account of mesoscopic fluctuations can be found in Refs. [14] and [15], respectively. 
mesoscopic samples which have been fabricated to the same specifications ${ }^{3}$ but differ in the positions of their defects. Accordingly, the persistent currents $I(\phi)$, that is the set $\left\{I_{m}\right)$ of its Fourier amplitudes, should be considered as stochastic quantities which are completely characterized if all correlation functions are known. Thus, we have to consider the set of all $k$-point correlation functions

$$
\begin{aligned}
& \mathscr{M}_{I}\left(m_{1}, \cdots, m_{k}\right)=\left\langle I_{m_{1}} I_{m_{2}} \cdots I_{m_{k}}\right\rangle_{c} \\
& \mathscr{M}_{I}\left(\phi_{1}, \cdots, \phi_{k}\right)=\left\langle I\left(\phi_{1}\right) I\left(\phi_{2}\right) \cdots I\left(\phi_{k}\right)\right\rangle_{c}
\end{aligned}
$$

where the angular brackets $\langle\cdots\rangle$ mean the average with respect to the impurity ensemble introduced above. Specifically, $\langle\cdots\rangle_{c}$ means the cumulant correlation functions. For instance

$$
\left\langle I\left(\phi_{1}\right) I\left(\phi_{2}\right)\right\rangle_{c}=\left\langle I\left(\phi_{1}\right) I\left(\phi_{2}\right)\right\rangle-\left\langle I\left(\phi_{1}\right\rangle\left\langle I\left(\phi_{2}\right)\right\rangle\right.
$$

Note that in a graph theory, the cumulants are defined as the set of connected diagrams.

In the idealized situation where no magnetic field penetrates the metal ring, Eqs. (2.1), (2.2) contain the same information; in general, however, only Eq. (2.2) carries the full information.

As a rule, it is more convenient to calculate the correlation function of thermodynamic potentials and to calculate the currents by taking the derivative with respect to the fluxes as shown in Eq. (1.1). There are arguments, which show that the stochastic properties of the persistent currents are adequately obtained within the grand canonical ensemble. Thus, we introduce the following set of $k$-point correlation functions

$$
\mathscr{M}_{\Omega}\left(\phi_{1}, \cdots, \phi_{k}\right)=\left\langle\Omega\left(\phi_{1}\right) \Omega\left(\phi_{2}\right) \cdots \Omega\left(\phi_{k}\right)\right\rangle_{c}
$$

It is obvious - see Eq. (1.6) for instance - that the magnitude of the persistent currents may equally be characterized by energies. In this respect, one should recognize that a mesoscopic metal ring displays a variety of quite different energies as follows.

(i) Fermi energy: $\varepsilon_{F}$

(ii) Elastic scattering rate: $1 / \tau$

(iii) Energy to localize an electron in one half of the ring: $v_{F} / L$

(iv) Thouless energy: $E_{c}=D / L^{2}$

(v) Mean level spacing: $\Delta=\left(2 \mathscr{N}^{0} \mathscr{\eta}\right)^{-1}-\varepsilon_{F} / N$

where $\varepsilon_{F}$ and $v_{F}\left(=p_{F} / m\right)$ are energy and velocity of electrons at the Fermi level, and $\mathscr{N}^{0}=m p_{F} / 2 \pi^{2}$ is the density of states. The diffusion constant is $D=v_{F} l / 3$, where $l=v_{F} \tau$ is the mean free path. Furthermore, $L$ is the circumference and $\mathscr{V}$ the volume of the mesoscopic ring.

Theoretical models on persistent currents differ in whether the Coulomb interaction is taken into account or not. With non-interacting fermions, the problem is simplest for a perfect ring geometry $[1,2]$. Inclusion of impurity scattering does already lead to non-

${ }^{3}$ Micrographs of the samples reveal irregularities in their shapes. We will assume that these irregularities can effectively be simulated by random positions of the defects. Such an assumption is partially supported by concepts known from the theory of chaotic systems [16]. 
trivial complications. One may either resort to computer calculations [5]; to random matrix theory; to the diffuson/cooperon expansion [17] within the impurity technique for Green functions; or to the supersymmetry technique [18]. If one wishes to include the Coulomb interaction between the electrons, it seems to be unavoidable to make use of methods developed in quantum field theory $[17,19]$.

Of the hierarchy of correlation functions $(2.1,2)$, the average current $\langle I(\phi)\rangle$ (resp $\left.\left\langle I_{m}\right\rangle\right)$ and the two-point correlator $\left\langle I(\phi) I\left(\phi^{\prime}\right)\right\rangle_{c}$ (resp $\left.\left\langle I_{m} I_{m}\right\rangle_{c}\right)$ have been calculated repeatedly in the past. There seems to be a general agreement on the following properties:

$$
\begin{aligned}
& \left\langle I_{2 p+1}\right\rangle=0 \\
& \left\langle I_{m} I_{m^{\prime}}\right\rangle_{c}=0 \quad m \neq m^{\prime} .
\end{aligned}
$$

For a general orientation, we list below some results, by order of magnitude and for $T=0$.

(a) non-interacting fermions

(i) ballistic limit $(l \gg L)$; one channel ${ }^{4}$ only

$I_{m} \sim \pm e v_{F} / L$.

The sign above depends on the number of electrons.

(ii) diffusive limit $(I \ll L)$

$\left\langle I_{2 p}\right\rangle \sim \begin{cases}0 & \text { grand canonical ensemble } \\ e \Delta & \text { canonical ensemble }\end{cases}$

$\left\langle I_{m}^{2}\right\rangle_{c} \sim\left(e E_{c}\right)^{2}$

(b) interacting electrons; diffusive limit only

$$
\begin{aligned}
& \left\langle I_{2 p}\right\rangle \sim e E_{c} \\
& \left\langle I_{m}^{2}\right\rangle_{c} \sim\left(e v_{F} / L\right)^{2} .
\end{aligned}
$$

In our recent letter [11], we have also presented arguments which show that the Fourier components $I_{m}$ are independent Gaussian variables provided that the number $N$ of electrons in a ring is sufficiently large.

The two experimental observations reported so far contain different information. In the experiment of the Bell group [12], the persistent current of an ensemble of $\sim 10^{7}$ copper rings has been measured; this supplies information on the average current $\left\langle I_{m}\right\rangle$. The odd harmonics seems to be absent; otherwise the following value for $\left\langle I_{2}\right\rangle$ and other parameters have been reported:

\footnotetext{
${ }^{4}$ Usually, the number of transverse channels is taken to be equal to $p_{F}^{2} \mathscr{A} / \pi^{2}$, where $\mathscr{A}=\mathscr{h} L$ is the
} cross section of the ring. 


$$
\begin{array}{ll}
\left\langle I_{2}\right\rangle \sim 0.6 e E_{c} \\
L \sim 2 \cdot 10^{-4} \mathrm{~cm} \quad l / L \sim 1 / 70 \\
\Delta \sim 0.2 \mathrm{mK} \quad E_{c} \sim 25 \mathrm{mK} \quad v_{F} / L \sim 5 \mathrm{~K} .
\end{array}
$$

This agrees roughly with the theory for interacting electrons in the diffusive limit.

In the experiments of the IBM group [13], the persistent currents of three different single gold rings have been measured. In such a single ring experiment, one expects the observed current to be of the order ${ }^{5}\left\langle I_{m}^{2}\right\rangle_{c}^{1 / 2}$ (usually this is very much larger than the average current $\left.\left\langle I_{m}\right\rangle\right)$. The following values have been reported:

$$
\begin{array}{lll}
I_{1} \sim(0.3-2.0) e v_{F} / L \\
L \sim 8 \cdot 10^{-4} \mathrm{~cm} & l / L \sim 1 / 110 \\
\Delta \sim 0.02 \mathrm{mK} & E_{c} \sim 4 \mathrm{mK} & v_{F} / L \sim 1 \mathrm{~K} \\
\varepsilon_{F} \sim 10^{5} \mathrm{~K} & 1 / \tau \sim 10^{2} \mathrm{~K} & N \sim 10^{10} .
\end{array}
$$

The experimental result agrees roughly with a theory of interacting electrons which we have put forward [11]. Note, however, that the relation $\left.\left\langle I_{1}^{2}\right\rangle_{c}\right\rangle\left\langle I_{1}\right\rangle^{2}$ requires the sign of the observed currents to vary randomly from sample to sample (and also from Fourier component to Fourier component). Experimentally, the sign of the current has been positive for the two samples that have been checked; but obviously no conclusion can be drawn from such a limited sampling.

However, the argumentation in our letter [11] has been commented critically by various of our colleagues. Therefore, it is necessary to give an account how our theory can be put on a solid basis. This will be done in the following.

\section{Non-interacting electrons}

As already mentioned, the differences between the canonical and the grand canonical ensemble appear to be negligeable in the problem of stochastic fluctuations. Therefore we will take the grand canonical ensemble as it has been suggested in Eq. (2.4); and we will calculate the following two-point correlator:

$$
\mathscr{M}_{\Omega}\left(\phi, \phi^{\prime}\right)=\left\langle\Omega(\phi) \Omega\left(\phi^{\prime}\right)\right\rangle_{c}
$$

In the quantum field theory, the grand canonical potential and its correlation functions are represented graphically by vacuum diagrams. ${ }^{6}$ Specifically, in the impurity

\footnotetext{
${ }^{5}$ In the literature, this rms current is sometimes also called the typical current.

${ }^{6}$ See, for instance, $\S 15$ of Ref. [19]. Note, however, that the rules for the combinatorial factors of compact vacuum diagrams need reformulation. Accordingly, there is a factor of $M^{-1}$, where $M$ is the number of equivalent sites a Green function may have in a given diagram. - In order to check the combinatorics, one may calculate a physical response by taking the derivative with respect to an appropriate source field.
} 
technique for Green functions [17, 19], the flux dependence is obtained from the dominant diffuson and cooperon contributions (particle-hole and particle-particle ladders). Note that diffuson and cooperon contribute to the two-point correlation in a symmetric way, the flux dependence being $(\phi-\phi)$ and $(\phi+\phi)$, respectively; hence in all diagrams for $\mathscr{H}_{\Omega}(\phi, \phi)$, we omit the arrows on the electronic loops implying that both senses of circulation have to be considered.

For non-interacting electrons, the relevant expression is shown in Fig. 1 where, by symmetry of the graph, the term with $M$ connecting impurity lines has the non-trivial combinatorial factor $M^{-1}$. Hence a typical term is given by $(x \equiv(r, \tau)$ comprises space and time)

$$
\begin{array}{r}
\frac{1}{M}\left(2 \pi \mathscr{N}^{0}\right)^{-M} \int d x_{1} \ldots d x_{M} \Gamma^{G}\left(x_{1}-x_{1}^{\prime}\right) \mathscr{G}\left(x_{1}, x_{2}\right) \mathscr{G}\left(x_{1}^{\prime}, x_{2}^{\prime}\right) \\
\ldots \Gamma^{G}\left(x_{M}-x_{M}^{\prime}\right) \mathscr{G}\left(x_{M}, x_{1}\right) \mathscr{G}\left(x_{M}^{\prime}, x_{1}^{\prime}\right)
\end{array}
$$

where we have associated $\Gamma^{G}\left(x_{i}-x_{i}^{\prime}\right) / 2 \pi \mathscr{N}^{0}$ with each impurity line connecting $\Omega(\phi)$ with $\Omega\left(\phi^{\prime}\right)$, represented in Fig. 1 by the two electronic loops. Anticipating arguments to be presented in the next chapter, we distinguish in the impurity scattering the rates $\Gamma^{G}\left(G=\right.$ gain; scattering in) and $\Gamma^{L}(L=$ loss; scattering out), the latter entering the Green functions in (3.2) through self-energy insertions. We note that the series starts with $M=2$; the term with $M=1$ has to be omitted for reasons of charge neutrality.

Fig. 1 Two-point correlator $\mathscr{M}_{\Omega}(\phi, \phi)$ of the grand canonical potentials $\Omega(\phi)$ and $\Omega(\phi)$. For non-interacting electrons, each potential is represented by an electronic loop (full lines represent impurity averaged Green functions) carrying a flux as a labelled. The broken lines represent (correlated) impurity scattering events. Note the combinatorial factor $M^{-1}$ since the $M$ connecting impurity lines are undistinguishable. (In the diagram, $M=5$.) The impurity ladder (diffuson as well as cooperon) carries the momentum $\mathbf{q}$.

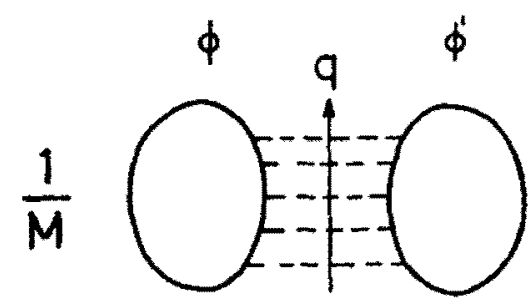

We consider now the above expression in momentum space. There, we encounter the expression

$$
I_{0}(\mathbf{q}, \omega)=\frac{\Gamma^{G}}{2 \pi \mathscr{N}^{0}} \int \frac{d^{3} k}{(2 \pi)^{3}} \mathscr{G}(\mathbf{k}, \varepsilon) \mathscr{G}(\mathbf{k}-\mathbf{q}, \varepsilon-\omega) .
$$

In standard approximation valid for $|\omega|, v_{F} q \ll \varepsilon_{F}$, and in the frequency regime $\varepsilon(\varepsilon-\omega)<0$, it is given by

$$
I_{0}(\mathbf{q}, \omega)=\frac{\Gamma^{G}}{v_{F} q} \operatorname{arctg}\left(\frac{v_{F} q}{|\omega|+\Gamma^{L}}\right) .
$$

Hence

$$
\mathscr{M}_{\Omega}=4 T \sum_{\omega, q} \frac{|\omega|}{2 \omega} \sum_{M=2}^{\infty} \frac{1}{M}\left[I_{0}(\mathbf{q}, \omega)\right]^{M}
$$


where a spin factor of four has been included. From this expression, the dominant flux dependence is extracted by considering the contribution from small $\omega$, $\mathbf{q}$, i.e. $|\omega| \tau<1$, $q l<1$, by noting that in this regime,

$$
1-I_{0}=\tau\left[|\omega|+\Gamma^{L}-\Gamma^{G}+D q^{2}\right]
$$

Above, we have assumed that $\Gamma^{L} \simeq \Gamma^{G} \simeq 1 / \tau$, but for illustration we have kept the explicit notation for the difference $\delta \Gamma=\Gamma^{L}-\Gamma^{G}$. Performing the $M$-summation in Eq. (3.5), we arrive at

$$
\mathscr{H}_{\Omega}=\frac{4 T}{2 \pi} \sum_{\omega, \mathrm{q}}|\omega|\left[\ln \left(\frac{1 / \tau}{|\omega|+\delta \Gamma+D q^{2}}\right)-I_{0}\right]
$$

where, for the ring geometry, $\mathbf{q}$ has to be taken one-dimensional such that

$$
\mathbf{q} \rightarrow \frac{2 \pi}{L}\left(n-\frac{\phi \pm \phi^{\prime}}{\phi_{0}}\right)
$$

with the upper and lower sign for the cooperon and diffuson, respectively.

For a technical detail, note that for any periodic function of the type

$$
X(\varphi)=\sum_{n=-\infty}^{+\infty} G\left(\left[D\left(\frac{2 \pi}{L}\right)^{2}\left(n-\frac{\varphi}{\phi_{0}}\right)^{2}\right]\right)=\sum_{m=-\infty}^{+\infty} e^{2 \pi i m \varphi / \phi_{0}} X_{m}
$$

the Fourier coefficients $X_{m}$ can be calculated according to

$$
X_{m}=\frac{1}{2 \pi} \int_{-\infty}^{+\infty} d x e^{-i m x} G\left(\left[E_{c} x^{2}\right]\right) .
$$

Since $X_{-m}=X_{m}$, only terms $\propto \cos 2 \pi m \varphi / \phi_{0}$ survive the summation in Eq. (3.9).

Note that in this Fourier representation, the combined contribution of cooperon and diffuson,

$$
\cos 2 \pi m \frac{\phi+\phi^{\prime}}{\phi_{0}}+\cos 2 \pi m \frac{\phi-\phi^{\prime}}{\phi_{0}}=2 \cos 2 \pi m \frac{\phi}{\phi_{0}} \cos 2 \pi m \frac{\phi^{\prime}}{\phi_{0}}
$$

leads to a result where the thermodynamic potentials are even functions of the fluxes, as it should be.

In the further calculation, we put $\delta \Gamma=\Gamma^{L}-r^{G}=0$, which is true for noninteracting electrons. Also, we recognize that a substantial contribution to the Fourier coefficients of the type (3.10) is connected with the cooperon/diffuson singularity at $\omega=$ $q=0$ (which is not affected by the omission of a finite number of terms in the series of Eq. (3.5)).

Eventually, we obtain the result (see also Ref. [10])

$$
\begin{aligned}
& \left\langle I_{m} I_{m}\right\rangle_{c}=\delta_{m m^{\prime}}\left\langle I_{m}^{2}\right\rangle_{c} \\
& \left\langle I_{m}^{2}\right\rangle_{c}=\frac{1}{m^{3}} \frac{96}{\pi^{2}}\left(e E_{c}\right)^{2}
\end{aligned}
$$

which has already been outlined in Sect. 2 . 


\section{Interacting electrons}

As shown by Ambegaokar and Eckern [6], the Coulomb interaction enhances the average persistent current considerably above ${ }^{7}$ the value for non-interacting fermions.

A characteristic feature of a Coulomb system is the rearrangement of charges which leads to a screened Coulomb interaction $\tilde{u}(k)$ (see the diagram of Fig. 2) which is given in reasonable approximation by

$$
\tilde{u}(k)=\frac{4 \pi e^{2}}{k^{2}+8 \pi e^{2} \mathscr{N}^{0}} .
$$

Fig. 2 The screened Coulomb interaction is represented by a double wavy line. The simple wavy line is the bare Coulomb interaction.

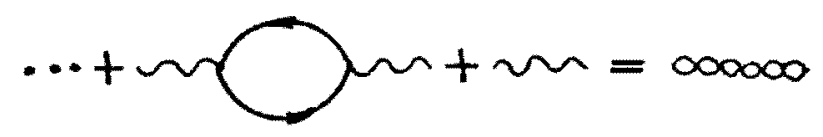

The theory of Ref. [6] is based on the Hartree-Fock approximation. Since the Fock contribution introduces only a correction factor of order 1 (e.g. $\frac{1}{2}$ ), we will neglect Fock contributions and consider Hartree diagrams only in the following.

Quite generally, Hartree diagrams consist of electronic loops which are connected by bare Coulomb interactions $u(k)=4 \pi e^{2} / k^{2}$ in a branching (simply connected) type of topology. As a rule, strings of fermionic loops can be put together to yield a screened Coulomb interaction $\tilde{u}(k)$. For illustration, the Hartree contribution to the impurity averaged thermodynamic potential ${ }^{8}\langle\Omega(\phi)\rangle$ is shown in the diagram of Fig. 3. From there, one obtains

$$
\left\langle I_{m}\right\rangle=\frac{1}{m^{2}} \frac{8}{\pi} e E_{c}
$$

In our letter [11] on the stochastic fluctuations of persistent currents, we have considered a simple Hartree-type contribution to the correlator $\left\langle\Omega(\phi) \Omega\left(\phi^{\prime}\right)\right\rangle$ as shown in

Fig. 3 Hartree diagram for the average grand canonical potential. Since both electronic loops carry the same flux, only the cooperon produces a flux sensitive contribution.

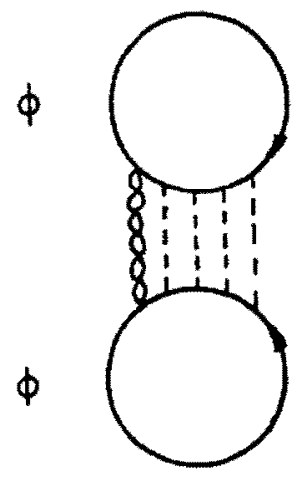

${ }^{7}$ A renormalization of the interaction constant $[6 \mathrm{~b}]$ reduces this enhancement to a less spectacular value.

${ }^{8}$ Note that with Coulomb interaction, there is no difference between the canonical and the grand canonical ensemble. 
Fig. 4a. In a different representation, one may introduce a pseudo-interaction $W(k)$ as defined in the diagram of Fig. $4 \mathrm{~b}$. Thus, the diagram of Fig. 4 a can be represented as shown in Fig. $4 \mathrm{c}$. As mentioned earlier, the formulation of our theory has been commented critically by various of our colleagues [20-22]. For instance, it has been observed that the pseudo-interaction should be considered rather a dressed impurity scattering [20], since it carries zero frequency only. Moreover, it has been observed [22] that the diagram of Fig. $4 \mathrm{~d}$ cancels exactly the one of Fig. $4 \mathrm{c}$.

a

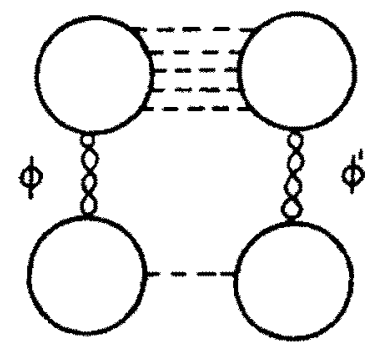

b

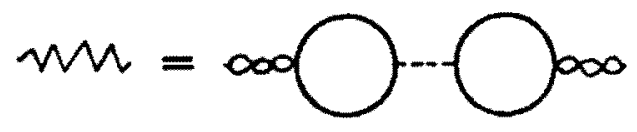

C

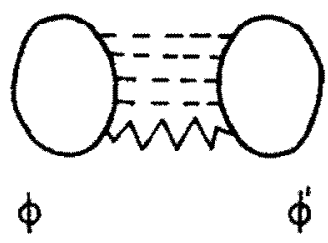

Fig. 4 (a) Hartree-type contribution to the two-point correlator $\mathscr{H}_{\Omega}(\phi, \phi)$. Each of the two Hartree diagrams consists of two electronic loops connected by a screened Coulomb interaction. Impurity lines between them are drawn to give a phase sensitive contribution to the correlator as large as possible. (b) The pseudo-interaction is shown as a jagged line. (c) Representation of diagram $4 \mathrm{a}$ in terms of the pseudo-interaction. (d) Self-energy insertion.

These few comments may serve as an illustration of the difficulties one encounters when trying to separate impurity scattering from Coulomb interaction. Note that these difficulties are most severe in the problem of stochastic fluctuations where interaction and impurity lines are complementary in their role of connecting various pieces of a diagram. Nevertheless, this problem provides also a good testing ground for developing a systematic theory since there, the various contributions can be discriminated and identified by their different dependence on the magnetic fluxes.

\section{Systematic theory of impurity scattering and Coulomb interaction}

In order to develop a consistent theory, we find it advantageous to consider an impurity as a defect of charge $z e$ which interacts with the electron by a bare Coulomb interaction, which is then screened in the standard way. For ease in drawing diagrams, we will henceforth represent a screened Coulomb interaction (previously a double wavy 
Fig. 5 (a) Screened Coulomb interaction. The broken line replaces the double wavy line of Fig. 2. (b) A heavy dot represents the factor $n_{i} z^{2}$. (c) This impurity line stands for the analytical expression $\tilde{u}(k) \cdot n_{i} z^{2} \cdot \tilde{u}(k)$.

line) by a dashed line (Fig. 5 a). Furthermore, a heavy dot (Fig. 5 b) means a factor $n_{i} z^{2}$, where $n_{i}$ is the density of the defects. Thus, an impurity line is represented consistently by two screened Coulomb lines attached to a heavy dot as shown in Fig. $5 \mathrm{c}$; its analytical expression is $\tilde{u}^{2}(k) n_{i} z^{2}$.

For convenience, we consider the limit of many defects of small charge, i.e. $n_{i} \rightarrow \infty$, $z \rightarrow 0$, such that $n_{i} z^{2}$ is finite. Then, a heavy dot has only two interaction lines attached to it.

We recognize the following elements of any impurity averaged Hartree diagram:

(i) screened Coulomb interaction;

(ii) electronic loops with at least three screened interactions attached to it;

(iii) impurity dots with exactly two screened interactions attached to it.

The additional specification (ii) can be understood as follows. A loop with only one interaction line can be interpreted as a Hartree self-energy contribution which can be absorbed by a proper renormalisation of the electronic chemical potential. Furthermore, a loop with only two interaction lines would imply a kind of screening of a screened interaction (formally: multiple counting of diagrams); obviously, it has to be omitted. ${ }^{9}$

In a conventional theory, the diagrammatic rules for the construction of the $k$-point correlation function $\mathscr{M}_{\Omega}\left(\phi_{1}, \cdots, \phi_{k}\right)$ for the grand canonical potential $\Omega$ as introduced in Eq. (2.4) are as follows:

(i) choose an appropriate class of vacuum diagrams for $\Omega$ in accordance with the requirements of the problem;

(ii) draw $k$ replicas of this class and label the replicas by their magnetic fluxes;

(iii) connect the replicas by impurity lines in all possible ways. It is also understood that each replica has impurity insertions where necessary.

The above rules imply that the various parts of one replica have to be connected by interaction lines (and not by impurity lines only), and that no interaction line is allowed to connect different replicas.

Within the present approach, these rules amount to about the following. Any diagram contributing to $\mathscr{M}_{\Omega}$ is fully connected and consists of $k$ subsections which are labelled by the fluxes $\phi_{k}$. Each subsection is completely separated from the remainder by heavy dots. Any subsection has to be connected within itself by interaction lines without a dot.

Having these general rules in our minds, we continue with a detailed discussion. Obviously, an elastic scattering rate $1 / \tau$ can be defined in the usual way,

$$
1 / 2 \pi \mathscr{N}^{0} \tau=n_{i} z^{2}\left\langle\left|\tilde{u}\left(\mathbf{p}-\mathbf{p}^{\prime}\right)\right|^{2}\right\rangle_{F S}
$$

\footnotetext{
${ }^{9}$ Evidently, this rule eliminates all the diagrams of Fig. 4.
} 
where $\langle\cdots\rangle_{F S}$ means the average with respect to the Fermi surface. Though not directly justifiable at metallic densities, in the following we will neglect the momentum dependence of $\tilde{u}(k)$ which is formally done by letting ${ }^{10} e^{2} \rightarrow \infty$ in Eq. (4.1).

In the following theory of stochastic fluctuations of phase sensitive quantities, the concept of a phase sensitive impurity line (PSI) is most important. The PSI is represented - see Fig. 6 - by a full diamond with two interaction lines attached to it. Its definition is given in the diagram of Fig. $6 \mathrm{a}$, and one should observe that the impurity ladder is built from PSI lines of which there are at least two. Note that the electronic selfenergy (elastic scattering rate) has to be calculated consistently with the PSI line as shown in Fig. 6b. If necessary, angular averages of the type shown in Eq. (5.1) are implied; and the analytical expression of a PSI will be denoted $\Gamma / 2 \pi \mathcal{N}^{0}$.

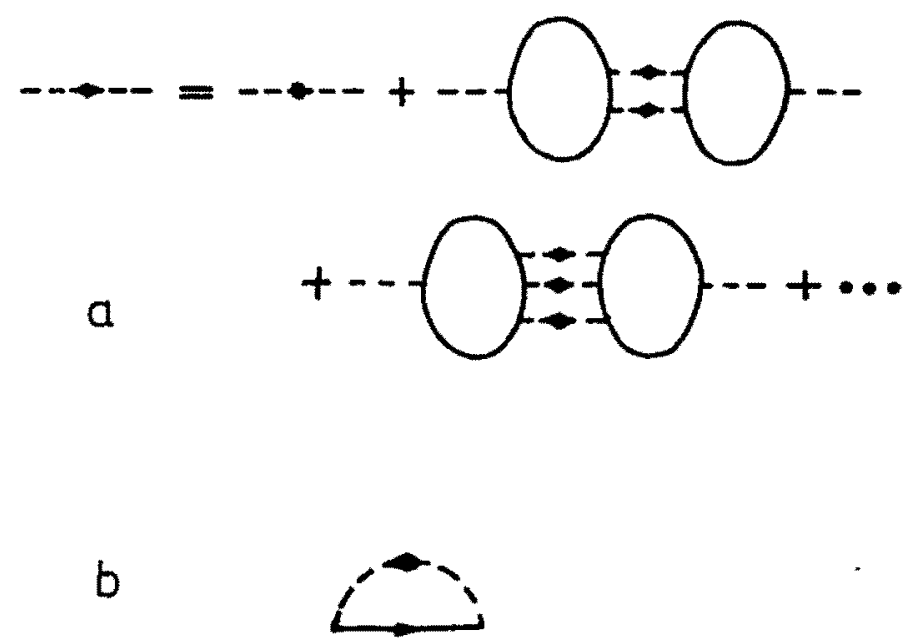

Fig. 6 (a) Definition of the phase sensitive impurity (PSI) line, which is represented by a full diamond with two screened interactions attached to it. Note that the pair of electronic loops include both senses of circulation (diffuson and cooperon). (b) Electronic self-energy.

In order to solve this non-linear equation in an appropriate way, we separate the qintegration of the impurity ladder in a regular $(q l \geq 1 ; r)$ and in a singular $(q l \leq 1 ; s)$ contribution. Similarly, we separate also the elastic scattering rate in a regular and a singular contribution, $\Gamma=\Gamma_{r}+\Gamma_{s}$, where the singular contribution is flux sensitive. Also, it will turn out that $\Gamma_{s}$ is very small; this fact as well as the condition $\Gamma_{r} / \varepsilon_{F} \ll 1$ allows us to calculate the leading part of the phase sensitive contribution from the impurity ladder in the range $q l \leqslant 1$ with impurity lines corresponding to the analytical expression $\Gamma_{r} / 2 \pi \mathscr{N}^{0}$.

As for the regular contribution, it is apparent that these diagrams merely lead to a renormalization of the scattering rate. For example, the diagram of Fig. 6a suggests that ${ }^{11}$

${ }^{10}$ In terms of the electronic density parameter $r_{s}$, this limit means $r_{s} \geqslant 10$ which is not realized in actual metals. It is expected that anisotropic impurity scattering will lead only to correction factors of order unity.

11 For $q l \geqslant 1$, one has roughly a factor $\Gamma_{r} / \varepsilon_{F}$ for each element of the ladder. Particle-hole and particle-particle ladder contributions lead to an extra factor 2. 


$$
\left.\Gamma_{r} \sim \frac{1}{\tau}+2\left\langle 2 \mathscr{N}^{0} \tilde{u}\right)^{2}\right\rangle_{F S} \Gamma_{r} \frac{\Gamma_{r} / \varepsilon_{F}}{1-\Gamma_{r} / \varepsilon_{F}}
$$

Of course, we do not claim that (5.2) is a reasonable approximation to calculate the Coulomb correction to the elastic scattering rate, which has to be taken anyhow from the experiment; instead we wish to emphasize that all regular contributions cancel in the difference $\Gamma^{L}-\Gamma^{G}$, whereas the singular contributions to $\Gamma^{L}-\Gamma^{G}$ remain finite due to the differences in the applied fluxes. Specifically, we have

$$
\Gamma^{L}-\Gamma^{G}=\frac{1}{2}\left[\Gamma_{s}(\phi, \phi)+\Gamma_{s}\left(\phi^{\prime}, \phi^{\prime}\right)\right]-\Gamma_{s}\left(\phi, \phi^{\prime}\right) \text {. }
$$

As mentioned above, $\Gamma_{s}$ can be obtained by evaluating the impurity ladder in the diagram of Fig. 6 for $q l \leqslant 1$ with a renormalized scattering rate $\Gamma_{r} \rightarrow 1 / \tau$. Thus one obtains $^{12}$

$$
\Gamma_{s}(k)=\left[2 \mathscr{N}^{0} \tilde{u}(k)\right]^{2} \frac{2 \tau}{\mathscr{N}^{0} \mathscr{V}} \frac{\operatorname{arctg} k l}{k l} T \sum_{\omega, q} \frac{|\omega|}{|\omega|+D q^{2}}
$$

Note that in the limit of isotropic impurity scattering $\left(e^{2} \rightarrow \infty\right)$ that we are considering here, one may replace $2 \mathscr{N}^{0} \tilde{u}(k)$ by 1 . Anticipating further developments, we have retained an essential momentum dependence in the singular part of the scattering rate.

The flux dependence of $\Gamma_{s}$ can be worked out following the rules laid down in Eqs. (3.8) and (3.10). The result will be expressed as follows:

$$
\Gamma_{s}=\frac{16 \tau}{\pi \mathscr{N}^{0} \mathscr{V}} \frac{\operatorname{arctg} k l}{k l} E_{c} \Psi(\phi, \phi)
$$

In the relation above,

$$
\Psi\left(\phi, \phi^{\prime}\right)=\sum_{m=1}^{\infty} \Psi_{m} \cos 2 \pi m \frac{\phi}{\phi_{0}} \cos 2 \pi m \frac{\phi^{\prime}}{\phi_{0}}
$$

where

$$
\begin{aligned}
& \Psi_{m}\left(T / E_{c}, \gamma / E_{c}\right)=\frac{1}{4}\left(\frac{2 \pi T}{E_{c}}\right)^{3 / 2} \sum_{n>0}\left(\frac{n \omega_{n}}{\tilde{\omega}_{n}}\right)^{1 / 2} \exp \left[-\left(\frac{m^{2} \tilde{\omega}_{n}}{E_{c}}\right)^{1 / 2}\right] \\
& \omega_{n}=2 \pi T n ; \quad \tilde{\omega}_{n}=\omega_{n}+\gamma .
\end{aligned}
$$

For sake of completeness, we have introduced ad hoc a phase-breaking rate $\gamma$ (the same for cooperon and diffuson). In the simple case $\gamma=T=0$,

$$
\Psi_{m}=m^{-3} .
$$

\footnotetext{
${ }^{12}$ It is also possible to estimate the contribution to $\Gamma_{s}$ from the region $q l \geqslant 1$. One finds that for the "true" $\Gamma_{s}$, the rhs of Eq. (5.4) should be multiplied by the factor $\left[1-\left[4 \mathscr{N}^{0} \tilde{u}(k)\right]^{2} \Gamma_{F} / \varepsilon_{F}\right]$. Usually $\Gamma_{r} / \varepsilon_{F} \ll 1$, which means that this factor is very close to one.
} 
Hence, for $k \sim k_{F}$, the flux dependent part of $\Gamma_{s}$ is in order of magnitude

$$
\Gamma_{s}-E_{c} / N
$$

which is a very small quantity $\left(\Gamma_{s} \ll 4\right)$.

For interacting electrons, the relevant contributions to the correlator $\mathscr{M}_{\Omega}(\phi, \phi)$ consist of the two parts which are shown diagrammatically ${ }^{13}$ in Fig. 7. Arguments which support this statement are given in the discussion of Sect. 7. We assert now, that the most important contribution to the current correlator $\mathscr{M}_{I}$ is obtained by differentiating the full diamonds with respect to $\phi$ and $\phi^{\prime}$. We mark the operation of taking this derivative by a dashed circle encompassing the object (see Fig. $8 \mathrm{a}$ ). Making use of the

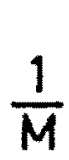

a

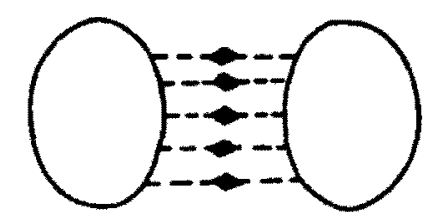

$b$

$\phi$<smiles>C1CCCCCCCCCCCC1</smiles>

a<smiles>C1CCCC23CCCCC2(CC1)C3</smiles>

$\phi$

b

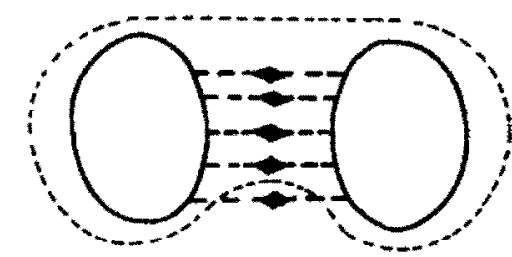

Fig. 7 Contributions to the correlator $\mathscr{M}_{\Omega}(\phi, \phi)$. (a) Ladder diagram with PSI lines $(M \geq 3)$. (b) A PSI line closed to a ring by a heavy dot.

Fig. 8 (a) Diagrammatic representation of the current correlator $\mathscr{H}_{I}(\phi, \phi)$. The dashed contour indicates the parts of the diagram which should be differentiated with respect to the fluxes $\phi$ and $\phi^{\prime}$. (b) The same as Fig. 8 with the definition of the PSI of Fig. $6 \mathrm{a}$ inserted. Again, the dashed contour indicates the parts with flux derivatives. (c) Representation of the correlator $\mathscr{M}_{\Omega}(\phi, \phi)$ as explained in the text.

13 Note that the ladder in the diagram of Fig. 7 a must have $M \geq 3$. 
diagrammatic definitions of Fig. 6 a, we recognize that $\mathscr{M}_{I}(\phi, \phi)$ can be represented by the ladder-type diagram as shown in Fig. $8 \mathrm{~b}$. Again, the dashed contour encloses those parts of the diagram the flux derivative should be operated on. Most important is the fact that one of the PSI lines is left out of this operation. Thus the equivalence of the $M$ PSI lines is broken, and there is no nontrivial combinatorial factor $M^{-1}$ in the diagram of Fig. $8 \mathrm{~b}$.

It is interesting to note that one might have equivalently well started with the diagram of Fig. $8 \mathrm{c}$ as representing the correlator $\mathscr{M}_{\Omega}(\phi, \phi)$; in fact, if one takes the flux derivative there, one obtains immediately the diagram of Fig. $8 \mathrm{a}$.

In the next section, it will be shown how to obtain an adequate expression for $\mathscr{M}_{\Omega}(\phi, \phi)$ from the diagram of Fig. 8c and from the relation (5.5) for $\Gamma_{s}$. Presently, we proceed following the presentation of Fig. $8 \mathrm{~b}$. Here, we will argue that most of the phase sensitive contribution is obtained when evaluating the ladder with $\Gamma$ replaced by $\Gamma_{r}$ in the range of $q l \leq 1$, as we have done when writing down Eq. (5.4). Letting $\Gamma_{r} \rightarrow 1 / \tau$ for simplicity, we conclude that $\mathscr{M}_{I}(\phi, \phi)$ can be obtained by proper differentiation of a correlator $\tilde{\mathscr{M}}_{\Omega}(\phi, \phi)$ shown in the diagram of Fig. $8 \mathrm{~b}$ with the dashed contour removed and with PSI lines replaced by simple impurity lines. Compare this diagram with the one of Fig. 1. Disregarding the rather trivial differences in the representation of the impurity lines (with/without heavy dot), one recognizes the essential difference between the diagrams of Fig. 1 and Fig. 8 b, which is in the combinatorial factors $M^{-1}$ and $M^{0}$, respectively.

Recall that the nontrivial combinatorial factor $M^{-1}$ in the diagram of Fig. 1 takes care of the fact that the $M$ impurity lines there are all equivalent, whereas in the diagram of Fig. 8 b, there is one impurity line which carries a distinct label ("color") but does not differ by a significant quantitative measure. This means that ${ }^{14}$ (cf. Eq. (3.5))

$$
\overline{\mathscr{M}}_{\Omega}(\phi, \phi)=4 T \sum_{\omega, \mathbf{q}} \frac{|\omega|}{2 \pi} \sum_{M=3}^{\infty}\left[I_{0}(\mathbf{q}, \omega)\right]^{M}=\frac{4 T}{2 \pi \tau} \sum_{\omega, \mathbf{q}} \frac{|\omega|}{|\omega|+D q^{2}} .
$$

Referring to Eqs. (5.4) and (5.5), we recognize that the above expression can be written as

$$
\tilde{\mathscr{M}}_{\Omega}(\phi, \phi)=\frac{16}{3 \pi^{2}}\left(\frac{v_{F}}{L}\right)^{2} \Psi(\phi, \phi)
$$

Consider now the current correlator. According to the relations derived above,

$$
\begin{aligned}
& \left\langle I_{m} I_{m}\right\rangle_{c}=\delta_{m m}\left\langle I_{m}^{2}\right\rangle_{c} \\
& \left\langle I_{m}^{2}\right\rangle_{c}=m^{2} \Psi_{m} \frac{16}{3 \pi^{2}}\left(\frac{e v_{F}}{L}\right)^{2} .
\end{aligned}
$$

If one wishes to compare this result with the one we have obtained in Ref. [11], one should consider the limit of isotropic impurity scattering $e^{2} \rightarrow \infty \quad\left(\alpha=8 \pi e^{2} \mathcal{N}^{0} / 2 k_{F}^{2}\right.$ $\rightarrow \infty$ there). This means that the pseudointeraction of Ref. [11],

\footnotetext{
${ }^{14}$ We are interested in the singular contribution. Thus, we may sum $\sum_{M}^{\infty}=1$.
} 


$$
\left\langle\pi \mathscr{N}^{0} W(\mathbf{k})\right\rangle_{F S} \equiv\left\langle\left[2 \mathscr{N}^{0} \tilde{u}(\mathbf{k})\right]^{2} / \tau\right\rangle_{F S}=\frac{\alpha}{\alpha+2} \frac{1}{\tau} \rightarrow \frac{1}{\tau}
$$

Hence the present approach confirms the result of Ref. [11].

\section{Higher order correlation functions and probability distribution}

In the following, we will show that for a sufficiently large number $N$ of electrons on the ring, the higher order (curnulant) correlation functions $\mathscr{M}_{\Omega}^{(k)}, k \geq 3$, are negligeably small compared to the two-point correlator $\mathscr{M}_{\Omega}^{(2)}$. If this is true, then it follows that the probability distribution is Gaussian. We will argue that for this higher order correlators, the Coulomb interaction does not enter in an essential way; therefore, the result will essentially also apply to the case of non-interacting electrons. Except for a correction factor, ${ }^{15}$ we will reproduce the results of our letter [11]. However, the concept of a phase sensitive impurity (PSI) line allows a more compact formulation.

As an introduction, consider first the diagram of Fig. $8 \mathrm{c}$ for the two-point correlator $\mathscr{A}_{\Omega}^{(2)}\left(\phi_{1}, \phi_{2}\right)$ in the form before the flux derivatives have been taken. Since $\Gamma=\Gamma_{r}+\Gamma_{s}$, and since $\Gamma_{s}\left(\phi_{1}, \phi_{2}\right) \ll \Gamma_{r}$ (which is independent of the fluxes), we recognize that we should select such contributions where the number of substitutions $\Gamma \rightarrow \Gamma_{s}$ is as small as possible. Of course the requirement will be that the final expression depends on all the fluxes; otherwise, its contribution to the current correlator would be zero.

Note that in the limit of isotropic scattering, $2 \mathscr{N}^{0} \tilde{u}(k) \rightarrow 1$, we have

$$
n_{i} z^{2} \rightarrow 2 \mathscr{N}^{0} / \pi \tau
$$

Within the substitution $\Gamma_{r} \rightarrow 1 / \tau$, the above expression can also be taken as the analytical expression for the regular part of a full diamond in the diagrams. Thus, the diagram of Fig. $8 \mathrm{c}$ corresponds to ${ }^{16}$

$$
\mathscr{H}_{\Omega}^{(2)}\left(\phi_{1}, \phi_{2}\right)=\mathscr{V} \int^{\prime} \frac{d^{3} k}{(2 \pi)^{3}} \frac{1}{\pi^{2} \tau} \Gamma_{s}\left(k ; \phi_{1}, \phi_{2}\right)
$$

where the $k$-integration is restricted to the range $k<2 k_{F}$. Inserting the expression (5.5) for $\Gamma_{s}$, one obtains

$$
\mathscr{\mathcal { M }}_{\Omega}^{(2)}\left(\phi_{1}, \phi_{2}\right)=R^{(2)}\left(\frac{v_{F}}{L}\right)^{2} \Psi\left(\phi_{1}, \phi_{2}\right)
$$

where

$$
R^{(2 p)}=\mathscr{V}_{r}^{(2 p)} \int^{\prime} \frac{d^{3} k}{(2 \pi)^{3}}\left[\frac{32}{9 \pi^{3}} \frac{1}{N} \frac{k_{F}}{k} \operatorname{arctg} k l\right]^{p}
$$

\footnotetext{
is We thank the authors of Ref. [21] for pointing out a mistake in our previous estimate [11] of $\mu_{\Omega}^{(k)}$ for $k$ odd (see below).

${ }^{16}$ We mark the correlation functions obtained from the prescription above by a bar.
} 
with $r^{(2)}=1$, and $r^{(2 p)}=(2 p-2) ! ! / 2$ for $p>1$. Since $k_{F} l \ll 1$, one obtains $R^{(2)}=$ $16 / 3 \pi^{2}$ in agreement with Eq. (5.10) (as already mentioned in Ref. [11] in connection what we called method (II) there).

Next, we consider even correlation functions $\mathscr{A}_{\Omega}^{(2 p)}\left(\phi_{1}, \cdots, \phi_{2 p}\right)$. For illustration, consider Fig. 9 a, where $p=3$. According to what has been said above, we obtain the largest phase sensitive contribution if we replace the full diamonds alternatively by $\Gamma_{s}$ and $\Gamma_{r}$ as we go around the ring. Thus, we obtain

$$
\overline{\mathscr{M}}_{\Omega}^{(2 p)}\left(\phi_{1}, \cdots, \phi_{2 p}\right)=R^{(2 p)}\left(\frac{v_{F}}{L}\right)^{2 p} \prod_{P}^{\prime} \Psi\left(\phi_{1}, \phi_{2}\right) \Psi\left(\phi_{3}, \phi_{4}\right) \cdots \Psi\left(\phi_{2 p-1}, \phi_{2 p}\right)
$$

where the product includes those permutations ${ }^{17}$ of the $2 p$ fluxes which lead to a different functional dependence. The prefactor $R^{(2 p)}$ has to be calculated according to Eq. (6.4). Explicitly,

$$
R^{(2 p)} \sim \frac{(2 p-2) ! !}{N^{p-1}} \begin{cases}1 & p<3 \\ \ln \left(k_{F} l\right) & p=3 \\ \left(k_{F} l\right)^{p-3} & p>3\end{cases}
$$

where the factor $\left(k_{F} l\right)^{p-3}$ reflects that the typical momentum going around the diagram of Fig. 9 a (compare Eq. (6.4)) is small, $k-l^{-1}$, for $p>3$. We recognize that $R^{(4)} / R^{(2)} \sim 1 / N$. Since $N \sim 10^{10}$ in the experiment of Ref. [13], this means that $\mathscr{A}_{\Omega}^{(4)}$, and all the even higher order correlation functions are negligeable.

Odd correlation functions require an additional discussion. If one were to follow, as we did in Ref. [11], the prescription explained above in the evaluation of the diagram shown in Fig. 9 a, one would realize that the alternating sequence of $\Gamma_{s}$ and $\Gamma_{r}$ does not

Fig. 9 (a) Diagrammatic representation of higher order correlation functions $\mathscr{k}^{(k)}(k=6$ in the figure). (b) Interaction of two neighboring impurity ladders in the third order correlation function.
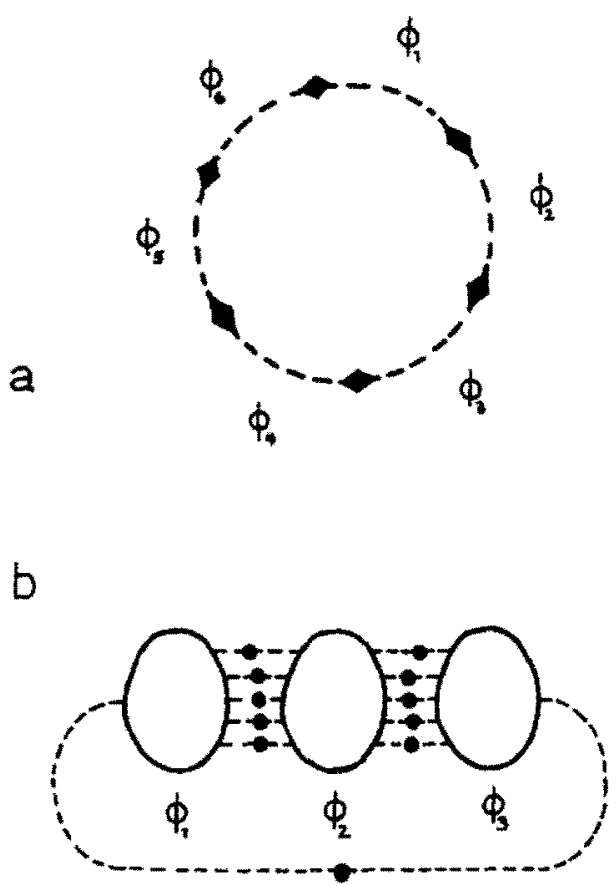

17 Presently, there are $(2 p-1) ! !$ different permutations. 
fit onto the ring: At one point, two $\Gamma_{s}$ will be close to each other, leading to a flux dependence which differs from Eq. $(6.5)$ such that the sequence of variables, $\{1,2 ; 3,4$; $\ldots 2 p-1,2 p\}$, is replaced by $\{1,2 ; 2,3 ; 4,5 ; \ldots 2 p, 2 p+1\}$. In order of magnitude, this contribution is $-N^{-p}(l / L)\left(k_{F} l\right)^{\max (p-2,0)}$.

However, as pointed out in Ref. [21], the odd correlations are dominated by an additional contribution, not obtainable within the above PSI scheme, which loosely speaking has two phase sensitive ladders next to each other, as shown for the example $k=3$ in Fig. 9b. We consider this three-loop term in more detail, with due consideration of the facts that (i) the total momentum along the ladders is small, and that (ii) the one extra impurity line carries a large momentum $\sim k_{F}$. Thus one obtains

$$
\mathscr{M}_{\Omega}^{(3)}\left(\phi_{1}, \phi_{2}, \phi_{3}\right)=R^{(3)}\left(\frac{v_{F}}{L}\right)^{3} \prod_{P}^{\prime} \Psi^{(3)}\left(\phi_{1}, \phi_{2}, \phi_{3}\right)
$$

where

$$
R^{(3)}-k_{F} L / N
$$

is considerably larger than our (above mentioned) previous estimate [11]. In Eq. (6.7), the function $\Psi^{(3)}\left(\phi_{1}, \phi_{2}, \phi_{3}\right)$ has the representation

$$
\begin{aligned}
& \Psi^{(3)}\left(\phi_{1}, \phi_{2}, \phi_{3}\right) \\
& \quad=\sum_{m, m^{\prime}=1}^{\infty} \Psi_{m, m^{\prime}}^{(3)} \cos 2 \pi m \frac{\phi_{1}}{\phi_{0}} \cos 2 \pi m \frac{\phi_{2}}{\phi_{0}} \cos 2 \pi m^{\prime} \frac{\phi_{2}}{\phi_{0}} \cos 2 \pi m^{\prime} \frac{\phi_{3}}{\phi_{0}}
\end{aligned}
$$

where $\Psi_{m, m}^{(3)}$, which is symmetric, for $\gamma=T=0$ is given by

$$
\Psi_{m, m^{\prime}}^{(3)}=\left[m m^{\prime}\left(m+m^{\prime}\right)^{2}\right]^{-1}
$$

The dominant phase sensitive contribution to correlation functions of order $2 p+1$ are found by combining a single three-loop element with the minimal number of simple ladders, of which there are $p-1$. Thus, we obtain

$$
\begin{aligned}
& \mathscr{M}_{\Omega}^{(2 p+1)}\left(\phi_{1}, \cdots, \phi_{2 p+1}\right) \\
& \quad=R^{(2 p+1)}\left(\frac{v_{F}}{L}\right)^{2 p+1} \prod_{p}^{\prime} \Psi^{(3)}\left(\phi_{1}, \phi_{2}, \phi_{3}\right) \Psi\left(\phi_{4}, \phi_{5}\right) \cdots \Psi\left(\phi_{2 p}, \phi_{2 p+1}\right)
\end{aligned}
$$

where $^{18}$

$$
R^{(2 p+1)}-\frac{k_{F} L}{N} R^{(2 p)}
$$

Note that in the experiment of Ref. [13], $k_{F} L / N \sim 10^{5} / 10^{10} \sim 10^{-5}$. Thus, we see that even in this unfavorable case, $\mathscr{M}_{\Omega}^{(3)}$ - and also the higher order correlations - can

${ }_{18}$ One should notice that $N / k_{F} L$ is about the number of transverse channels. 
be neglected in comparison with the two-point correlator $\mathscr{M}_{\Omega}^{(2)}$. If this is the case, we may conclude that the Fourier coefficients of the persistent currents $I_{m}$ are independent Gaussian variables with probability distribution

$$
P\left(\left(I_{m}\right)\right)=\text { const } \exp \left[-\sum_{m=1}^{\infty}\left(I_{m}-\left\langle I_{m}\right\rangle\right)^{2} / 2 \sigma_{m}^{2}\right]
$$

where $\sigma_{m}^{2}=\left\langle I_{m}^{2}\right\rangle_{c} \sim\left(e v_{F} / L\right)^{2}$, and where $\left|\left\langle I_{m}\right\rangle\right| \ll \sigma_{m}$ is negligeably small.

At this point, we wish to emphasize an important difference between the stochastic fluctuations of persistent currents and the Aharonov-Bohm effect in mesoscopic normal-metal rings $[14,15]$. In the latter case, one may expand the measured quantity (i.e. the conductance) in a Fourier series $\propto \cos \left[2 \pi m \phi / \phi_{0}+\gamma_{m}\right]$, in a way similar to Eq. (1.5). However, the phases $\gamma_{m}$ for different values of $m$ appear to be completely uncorrelated with no theoretical prediction available. Therefore, only the power spectrum (with respect to $\phi$ ) of the measured quantity is physically relevant; in fact, this is the central message of the so-called ergodic hypothesis. In contrast to that, the phase of the persistent current is uniquely fixed by the condition that the current be an odd function of $\phi$. In the present theory, this requirement is satisfied since the diffuson and the cooperon contribute to the correlators in a symmetric way.

\section{Discussion}

Considering the expressions (3.5-7) for the correlation function $\mathscr{M}_{\Omega}(\phi, \phi)$ of noninteracting fermions, one might characterize our previous approach [11] as a first order expansion with respect to a (regular) Coulomb correction $\delta \Gamma_{r}^{G} \sim O(1 / \tau)$ of the elastic scattering rate $\Gamma^{G}$. Correspondingly, one may consider the diagram of Fig. $4 \mathrm{c}$ to be the result of an expansion with respect to $\delta \Gamma_{r}^{L}$. Since $\delta \Gamma_{r}^{G}=\delta \Gamma_{r}^{L}$, both contributions have to cancel.

In contrast to our previous presentation, we have now avoided any separation of the various contributions to the impurity scattering rate. As an alternative, we have put forward a self-consistent theory which is based on the concept of a phase sensitive impurity (PSI) line. For instance, the electronic self-energy is included from the beginning selfconsistently; therefore, diagrams of the type as shown in Fig. $4 \mathrm{~d}$ are already taken into account.

Specifically, we have proposed to calculate the correlation function $\mathscr{M}_{\Omega}\left(\phi \phi^{\prime}\right)$ following the diagrams of Fig. 7. Note that the contribution of the diagram $7 \mathrm{a}$ is about a factor $\left(\Gamma_{r} / \varepsilon_{F}\right)$ smaller than our final result, due to the condition $M \geq 3$. Thus, most of the contribution is from the diagram $7 b$.

The argument why this diagram should be included in this form is as follows. It is certainly true that the diagram shown in Fig. 10a contributes to the correlator a large amount but this contribution is not phase sensitive. Therefore, we have introduced its appropriate extension in the diagram of Fig. 7 b. In order to clarify its structure, we have redrawn this diagram in a detailed form as shown in Fig. 10 b.

At that point, one might argue that one should include simple impurity lines in all possible ways in this diagram. At the end of this process, a new PSI would emerge as shown by the substitution of Fig. 10c. However, such a new PSI is ruled out by the definition of Fig. 6: two simple impurity lines would appear in a way as representing two different processes. 


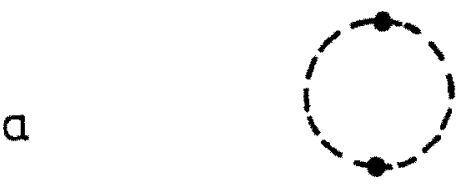

b

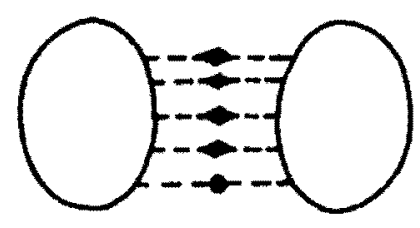

C

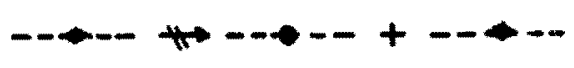

Fig. 10 Contributions to the correlator $\mathscr{M}_{\Omega}(\phi, \phi)$. (a) The largest contribution is phase insensitive. (b) Detailed structure of the phase sensitive contribution introduced by Fig. 7 b. (c) This substitution of the PSI is forbidden.

The phase sensitive contribution to the impurity scattering is very small, $\Gamma_{s} \sim E_{c} / N$, which may be about $10^{-16} \mathrm{eV} \sim 10^{-12} \mathrm{~K}$ in the experiment of Ref. [13]. This allows effectively an expansion of the correlator $\mathscr{M}_{\Omega}(\phi, \phi)$ to first order in $\Gamma_{s}$. It is this expansion which removes the nontrivial combinatorial factor $M^{-1}$ (where $M$ is the number of impurity lines), a typical feature of the diagrammatic expansion of the grand canonical potential. For a physical interpretation, we identify the regular scattering rate $\Gamma_{r}$ with the rate $1 / \tau$, where $\tau$ is the mean time of free flight. Thus, the diagrams for non-interacting fermions (Fig. 1) and interacting electrons (Fig. 8 b) appear to have the same structure; however, and most important, there is a difference in the combinatorial factor, which is $M^{-1}$ versus $M^{0}$, respectively. With some hindsight, one may argue about the factor $M^{0}$ in the interacting theory, that one of the $M$ impurity lines carries a distinct label ("color"), but not a different numerical value. We may add that we expect also not much of a difference if one goes beyond the Hartree approximation which has been the basis of the considerations above.

Eventually, one may ask about possible consequences the PSI may have in other problems of Coulomb interaction in disordered conductors [17]. We expect them to be rather insignificant. In fact, we have already found it to be of less importance in higher order correlation functions for persistent currents.

As a further illustration, consider the average persistent current, where the flux $\phi$ in all parts of a diagram has the same value. There, the screened Coulomb interaction $\tilde{u}\left(k_{,} \omega\right)$ does certainly not depend on the flux for $k l \leqslant 1$ provided that the impurity corrections are done properly, whence $\Gamma^{L}=\Gamma^{G}$. For $k l \geqslant 1$ a flux dependence appears; but it seems that such a contribution can be incorporated in the standard diagrams [6] if one takes interaction corrections to the screened Coulomb line into account.

In summary, we find that the stochastic fluctuations of the persistent currents are considerably enhanced by the Coulomb interaction between the electrons. In the idealized situation (no magnetic field in the metal ring), the persistent current can be repre- 
sented as a Fourier sum with components $I_{m} \sin 2 \pi m \phi / \phi_{0}$. The amplitude $I_{m}$ is a stochastic function depending on the random position of the impurities with a mean value $\left\langle I_{m}\right\rangle$ almost zero. Using date of Ref. [13], we find

$$
\left\langle I_{1}^{2}\right\rangle^{1 / 2}=\sigma_{1} \sim 0.42 e v_{F} / L
$$

This value, and the period $2 \pi \hbar c / e$, are in good agreement with the experimental results obtained for three gold rings [13]. We expect the current to vary randomly in sign from ring to ring; and from one Fourier component to the other. Detailed data [13] are given for a $(1.4 \times 2.6) \mu \mathrm{m}^{2}$ loop $(L=8 \mu \mathrm{m})$; for this loop, we compute the characteristic temperature $3 E_{c} \sim 12 \mathrm{mK}$. Thus for $T \sim 7.6 \mathrm{mK}$, the second harmonic is suppressed by a factor $\sim 13$, consistent with its almost absence in the power spectrum in Fig. $2 \mathrm{~d}$ of Ref. [13]. Concerning the parity of the current and its derivatives, we feel that Fig. $2 \mathrm{~b}$ and $2 c$ are a fair illustration of $I(-\phi)=-I(\phi)$.

Finally, in order to develop an idea of the width of the dominant $2 \pi \hbar \mathrm{cle}$-peak in the power spectrum, we take into account magnetic field penetration into the metal ring (non-ideal, that is, the real situation) and consider a flux-dependent phase-breaking rate $\gamma(\phi)$, as for example arises from the finite transverse dimension $(-900 \AA)$ of the loop. For the quoted parameters, we find [6b] $\gamma_{H}=1 / \tau_{H}=\beta^{2}\left(\phi / \phi_{0}\right)^{2} E_{c}$ with $\beta=0.5$. Hence, we estimate the relative width (full width at half maximum) as $\beta / \pi \sim 0.16$ (experimentally it is about twice this value), indicating that field penetration gives a substantial contribution to the broadening. Clearly, more detailed studies of the lineshape will be most interesting.

We wish to thank all our colleagues for their critique, in particular Yuval Gefen for insisting on clarification. This work has been supported in part by the German Israeli Foundation (GIF) and by the Deutsche Forschungsgemeinschaft (SFB195).

\section{References}

[1] M. Büttiker, Y. Imry, R. Landauer, Phys. Lett. 96 A (1985) 365

[2] H.F. Cheung, Y. Gefen, E. K. Riedel, IBM J. Res. Develop. 32 (1988) 359

[3] O. Entin-Wohlman, Y. Gefen, Europhys. Lett. 8 (1989) 477

[4] H.F. Cheung, E. Riedel, Y. Gefen, Phys. Rev. Lett. 62 (1989) 587

[5] H. Bouchiat, G. Montambaux, J. Phys. (Paris) 50 (1989) 2695; G. Montambaux, H. Bouchiat, D. Sigeti, R. Friesner, Phys. Rev. B 42 (1990) 7647

[6a] V. Ambegaokar, U. Eckern, Phys. Rev. Lett. 65 (1990) 381

[6b] U. Eckern, Z. Phys. B 82 (1991) 393

[7] Y. Imry, in: Quantum coherence in mesoscopic systems, B. Kramer (ed.), NATO ASI Series B: Physics, Vol. 254, Plenum, New York 1991, p. 221

[8a] E. Akkermans, A. Auerbach, J.E. Avron, B. Shapiro, Phys. Rev. Lett. 66 (1991) 76

[8b] A. Schmid, Phys. Rev. Lett. 66 (1991) 80

[8c] F. von Oppen, E. K. Riedel, Phys. Rev. Lett. 66 (1991) 84

[8d] B. L. Altshuler, Y. Gefen, Y. Imry, Phys. Rev. Lett. 66 (1991) 88

[9a] K. B. Efetov, Phys. Rev. Lett. 66 (1991) 2794

[9b] A. Altland, S. Iida, A. Müller-Groeling, H.A. Weidenmuller, Europhys. Lett. 20 (1992) 155

[10] E. K. Riedel, F. von Oppen, preprint, May 1991

[11] U. Eckern, A. Schmid, Europhys. Lett. 18 (1992) 457

[12] L. P. Levy, G. Dolan, J. Dunsmuir, H. Bouchiat, Phys. Rev. Lett. 64 (1990) 2074; L. P. Levy, Physica B 169 (1991) 245 
[13] V. Chandrasekhar, R. A. Webb, M. J. Brady, M. B. Ketchen, W. J. Gallagher, A. Kleinsasser, Phys. Rev. Lett. 67 (1991) 3578

[14a] B.L. Altshuler, Pis'ma Zh. Eksp. Teor. Fiz, 41 (1985) 530 [JETP Lett. 41, (1985) 648]

[14b] P. A. Lee, A.D. Stone, Phys. Rev. Lett. 55 (1985) 1622

[14c] A.D. Stone, X. Imry, Phys. Rev. Lett. 56 (1986) 189

[15] R.A. Webb, S. Washburn, C.P. Umbach, R. B. Laibowitz, Phys. Rev. Lett. 54 (1985) 2696; S. Washburn, R. A. Webb, Adv. Phys. 35 (1986) 375

[16] A. Szafer, B.L. Altshuler, Universal correlations in the spectra of chaotic systems with an A-B flux, Phys. Rev. Lett. 70 (1993) 587

[17 a] B.L. Altshuler, A.G. Aronov, D.E. Khmelnitskii, A. L. Larkin, in: Quantum theory of solids. I. M. Lifshitz (ed.), MIR Publishers, Moscow 1980, p. 130

[17b] B.L. Altshuler, A.G. Aronov, in: Electron-electron interactions in disordered systems, A. L. Efros, M. Pollak (eds.), North-Holland, Amsterdam 1985, p. 1

[18 a] K. B. Efetov, Adv. Phys. 32 (1983) 53

[18b] J. J.M. Verbaarschot, H. A. Weidenmüller, M. R. Zirnbauer, Phys. Rep. 129 (1985) 367

[19] A. A. Abriskosov, L. P. Gorkov, I. Ye. Dzyaloshinskii, Quantum field theoretical methods in statistical physics, Pergamon Press, Oxford 1965

[20] A.M. Finkelshtein, A.I. Larkin, private communication

[21] R.A. Smith, V. Ambegaokar, Europhys. Lett. 20 (1992) 161; and private communication

[22] N. Argaman, A. Altland, Y. Gefen, private communication 